\title{
O CANTEIRO DE OBRAS BRASILEIRO E A ADOÇÃO DE RECURSOS TECNOLÓGICOS - EM BUSCA DE MELHORIA DAS PRÁTICAS ATRAVÉS DA INOVAÇÃO DOS PROCESSOS
}

\section{The Brazilian Construction Site and Technological Resources Adoption - Searching of Better Practices through Process Innovation Márcia Regina de Freitas ${ }^{1}$}

Recebido em 19 de agosto de 2014; recebido para revisão em 27 de outubro de 2014; aceito em 23 de abril de 2015; disponível on-line em 15 de julho de 2015.

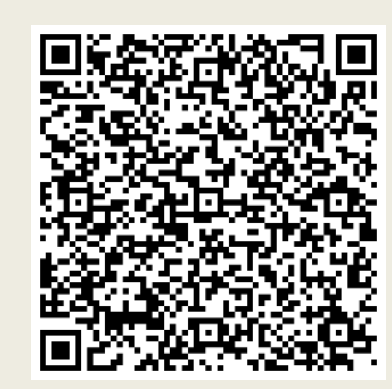

PALAVRAS CHAVE:

Construção Civil;

Inovação Tecnológica;

Canteiro de Obras.

KEYWORDS:

Civil Construction;

Innovation;

Construction Site.
RESUMO: Ações têm sido conduzidas por agentes setoriais para embasar os rumos a serem seguidos no processo de modernização da construção. A inserção de inovações tem se dado de forma ampla, considerando-se as peculiaridades do setor e as necessidades do mercado. É nítida, no entanto, a necessidade de entendimento do que é inovar, para encontrar formas possíveis de se adotar estratégias direcionadas à inserção de inovações. Este artigo apresenta recursos de Tecnologia da Informação (TI) avançados para uso nas atividades de construção, com alguns exemplos de aplicação. O objetivo principal deste artigo é somar esforços para consolidar a adoção e uso de recursos inovadores em obras, visando uma mudança na cultura por parte do setor, para absorção de novos recursos tecnológicos inovadores.

\begin{abstract}
Actions have been conducted by sector agents to give support to the companies in following the construction modernization process. The insertion of innovations has been broad considering the peculiarities of the industry and the needs of the market. It is clear, however, that is necessary to understand what is to innovate for find possible adoption strategies aiming at the inclusion of innovations in the process. This article provides shows research and published reports on the subject and presents features of advanced Information Technology (IT) for use in construction activities, with some application examples. The main objective of this article is joining efforts to consolidate the adoption and use of innovative resources in construction and to change sector culture in absorbing new innovative technological resources.
\end{abstract}

\footnotetext{
* Contato com os autores:

${ }^{1}$ e-mail : marciar.f@ig.com.br ( M. R. Freitas )

Doutora, Docente, Faculdade de Engenharia de Guaratinguetá.
}

\section{INTRODUÇÃO}

Devido a uma conjunção de ações tomadas em vários níveis, a construção civil (CC) brasileira tem passado por mudanças globais no sentido de fomentar as inovações relacionadas ao contexto de edificações. Com isso, a indústria da construção tem vivenciado, há alguns anos, um momento onde se juntou a demanda por moradia

NBR 15575 (ABNT, 2013) e seus requisitos gerais e específicos vieram nortear as empresas de inerente ao setor e o crescente acesso da população ao crédito, por meio de programas governamentais ou privados. Várias adequações no setor têm ocorrido em relação aos conceitos de desempenho, sustentabilidade ambiental de edifícios e Building Information Modeling (BIM) (BECERIK-GERBER e KENSEK, 2010), que são temas recorrentes ao se tratar de construção de Edificações. Em relação a desempenho, a norma como as empresas construtoras estão se adequando ao uso das tecnologias para 
construção em relação a uma gama de adequações, visando ajustes em seus processos de concepção e produção. Quanto à sustentabilidade nas edificações, este tem sido um tema cada vez mais discutido e com necessidade de estudo e aplicação efetiva. Finalmente, a etapa de concepção por meio da modelagem utilizando-se BIM que tem como conceito central favorecer a integração dos agentes e ações em projetos, tem sido visto como solução para problemas persistentes do setor (PAULA, UECHI e MELHADO, 2013; BALDAUF, FORMOSO e MIRON, 2013). Com esta trinca de conceitos a serem absorvidos pela indústria da construção, as empresas têm sido levadas a buscar alternativas, visando sua margem de lucros, prevendo uma redução de custo, aumento de produtividade e incremento de soluções tecnológicas e gerenciais (KRAINER et al., 2013).

Apesar dos esforços e do sucesso quanto à adoção de novas tecnologias no desenvolvimento de projetos, persistem dificuldades de novos recursos tecnológicos chegarem aos canteiros de obras, dificilmente ultrapassando os muros dos escritórios. A ideia central é, também nestes ambientes, absorver recursos de TIC (Tecnologia da Informação e Comunicação), como: troca de informações via redes de intranets, extranets e VPN (Virtual Private Network); comunicação sem fio (wireless) ou a cabo; etiquetagem com Rádio Frequência (RFID); uso de GPS (Global Positioning System), laser scanner; smartphones e tablets, etc.

Este artigo é um convite à avaliação do que existe atualmente em inovações tecnológicas para canteiros de obras e auxiliar no conhecimento para adequação quanto à absorção de tecnologia avançada.

\section{INOVANDO O CANTEIRO POR MEIO DE ALTA TECNOLOGIA - CHEGAREMOS LÁ?}

Ao que se refere ao uso de ferramentas de $\mathrm{TI}, \quad$ o artigo "Canteiro digital" (TÉCHNE, 2012) mostra melhorarem seus processos e produtos. Exceto pelas dificuldades de infraestrutura que independem do setor, tal como a disponibilidade e a velocidade de acesso à Internet nos canteiros, aspecto que tem evoluído timidamente, porém de forma constante, estas empresas tem se adaptado ao uso de novos equipamentos e tecnologias aplicadas aos canteiros de obras. Observa-se, porém, que não basta a simples adoção de recursos de TI sem que haja modelos eficientes de gestão da informação, integração entre dispositivos, capacitação dos usuários (técnicos e mão de obra) e especificação de tecnologias adequadas a necessidades específicas de cada canteiro e obra.

\subsection{RECURSOS PARA VISUALIZAÇÃO EM CANTEIROS - REALIDADE VIRTUAL E REALIDADE AUMENTADA}

Realidade Virtual (RV) é uma tecnologia definida como sendo um meio composto por simulações interativas em computador para perceber a posição e as ações do usuário e substituir ou aumentar a retroalimentação a um ou mais sentidos, favorecendo a sensação de estar mentalmente imerso ou presente na simulação (SHERMAN e CRAIG, 2003). Em síntese, por meio da $\mathrm{RV}$ define-se um mundo virtual onde o usuário poderá interagir com seu conteúdo, utilizando seus sentidos humanos como se estivesse em uma realidade paralela criada em computador. Esta tecnologia já vem sendo utilizada na construção civil por profissionais de arquitetura para a elaboração de projetos, treinamento de profissionais e como ferramenta de divulgação e venda de imóveis. Além de arquitetura, há possibilidades de utilização de RV em construção, visando diminuir as dificuldades de comunicação entre os parceiros envolvidos em um processo construtivo (KÄHKÖNEN, 2003). Apesar das vantagens na visualização dos processos, o usuário pode apresentar dificuldades no uso desta tecnologia pela necessidade de treino para familiarização com o uso de dispositivos não convencionais, o que pode ser um fator limitador 
no início. Há também a questão do elevado custo dos equipamentos e de sua sensibilidade para serem levados ao canteiro de obras.

A Realidade Aumentada (RA) é uma tecnologia que usa a RV para adicionar objetos virtuais à visualização de ambientes reais, em tempo real, utilizando-se de algum dispositivo tecnológico. É uma tecnologia que complementa o mundo real, sobrepondo ou compondo objetos virtuais a sua visualização, usada para facilitar a obtenção de informações imperceptíveis aos sentidos humanos (AZUMA, 1997; SHERMAN e CRAIG, 2003). Por meio da RA sobrepõem-se imagens geradas em computador com as informações do mundo real que é, então, observado por um indivíduo através de algum equipamento auxiliar (BEHZADAN, TIMM e KAMAT, 2008). Dessa maneira, eliminam-se as dificuldades, uma vez que adicionando os objetos virtuais (gráficos representativos de dados) à visualização, não se perde a noção do ambiente real, podendo usar mãos para mover e atuar sobre os gráficos (KIRNER et al., 2004), que estarão livres quando se utiliza óculos ou Head Mounted Displays (HMDs). Quanto a sua viabilidade de aplicação, atualmente a RA é possível por meio de smartphones e tablets que são dispositivos atualmente portados pela maiorias dos profissionais e encontrados em grande escala nos canteiros de empresas de médio a grande porte (TECHNE, 2012).

Um exemplo prático de uso de RA em construções é mostrado pela empresa Bechtel que utiliza o aplicativo de RA móvel Junaio (Metaio Inc.) em conjunto com o software Autodesk 360 Mobile, para sobrepor esquemas virtuais de sistemas mecânicos, elétricos e hidráulicos sobre as imagens atuais da construção (APPLE, 2013). Para tanto, utiliza o posicionamento por georeferenciamento para mapear a localização onde devem ser inseridos os modelos virtuais no ambiente físico (Figura 1).

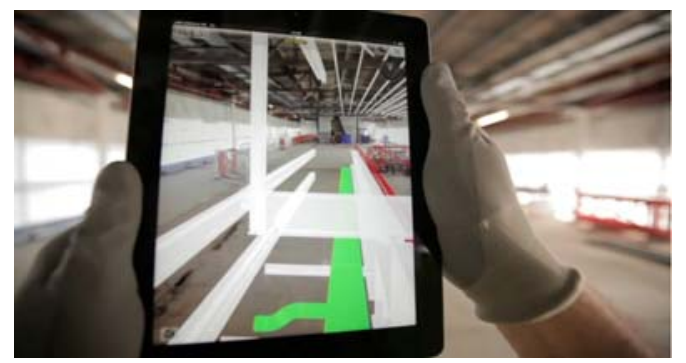

FIGURA 1: Esquemas virtuais de sistemas superpostos à construção usando RA com tablete.

FONTE: Captura de tela do vídeo disponível em Apple (2013).

Quanto aos aspectos construtivos a RA pode auxiliar na visualização de formas arquitetônicas e de resultados de simulação e análises de cenários, na fabricação de componentes estruturais ou na construção dos demais sistemas prediais (AMIM, 2007). De acordo com Freitas e Ruschel (2010), diversas pesquisas têm sido conduzidas no sentido de ampliar as aplicações da RA para a indústria de AECO (Arquitetura, Engenharia, Construção e Operação) pelo mundo. No Brasil, porém, são poucos os grupos que trabalham especificamente em aplicações direcionadas a este nicho ainda carente de modernas soluções para a melhoria de seus processos e produtos. De acordo com Azuma, Silvestri e Fabricio (2013), pesquisas realizadas com arquitetos, designers gráficos, construtoras, empresas de comunicação visual/marketing e imobiliárias, no estado do Paraná, são apontados como fatores que mais dificultam a adoção desta tecnologia pelo setor: custo de aquisição dos softwares, falta de investimento em tempo e disponibilidade concedidos aos colaboradores e profissionais técnicos para capacitação e treinamento, falta de mão de obra especializada no mercado para treinamento, entre outros.

\subsection{EQUIPAMENTOS PARA POSICIONAMENTO (SISTEMA GNSS OU GLOBAL NAVIGATION SATELLITE SYSTEM)}

O uso de técnicas matemáticas e computacionais para a interpretação e uso de informação geográfica é tratado na disciplina de conhecimento Geotecnologia ou 
geoprocessamento. $O$ tratamento de tais informações tem colaborado com atividades nas áreas de infraestrutura, gestão de recursos naturais, logística, transportes e segurança, comunicações, energia e planejamento urbano e regional, marketing, inteligência de negócios e mobilidade (NAKAMURA e GUIDARA JÚNIOR, 2010).

Para o posicionamento em canteiro de obras existem equipamentos que usam o Global Navigation Satellite System (GNSS), entre eles o GPS, usados por profissionais da área de topografia com a finalidade de facilitar o trabalho, reduzindo o tempo e aumentando a precisão nas marcações. 0 uso do GPS para a topografia tem sido considerado uma revolução, devido sua agilidade na determinação posições estáticas ou cinemáticas além da elevada precisão de alocação de coordenadas, impossíveis de se obter com métodos clássicos (NAKAMURA e GUIDARA JÚNIOR, 2010). Desta forma, observa-se cada vez mais o aumento do interesse por estas tecnologias espaciais pela possibilidade de obter alta acurácia nos posicionamentos com erros de pousos centímetros (ALVES, ABREU e SOUZA, 2013). A aplicação desta tecnologia na engenharia civil possibilita: verificações iniciais para checar elevações e áreas estimadas para projetos de canteiro, saneamento, rodovias e ferrovias; registro de corte e aterro em tempo real no canteiro com determinação de volumes de movimentação de terra; verificação e captura de informação para projeto as built (como construído), estaqueamento inclinado etc.

O uso desta tecnologia para a determinação de um posicionamento é feito por meio da trilateração, ou seja, determina-se a posição de um ponto requerido obtendo-se as distâncias que os separam dos satélites envolvidos na leitura e cujas posições no espaço são conhecidas. As distâncias são então calculadas pela determinação do tempo que os sinais de rádio demoram a atingir o receptor, partindo dos satélites. A Figura 2 mostra a utilização deste tipo de tecnologia para a locação em canteiro, na fase de pré-projeto, para a implantação da obra para posicionar os alicerces e fundações.

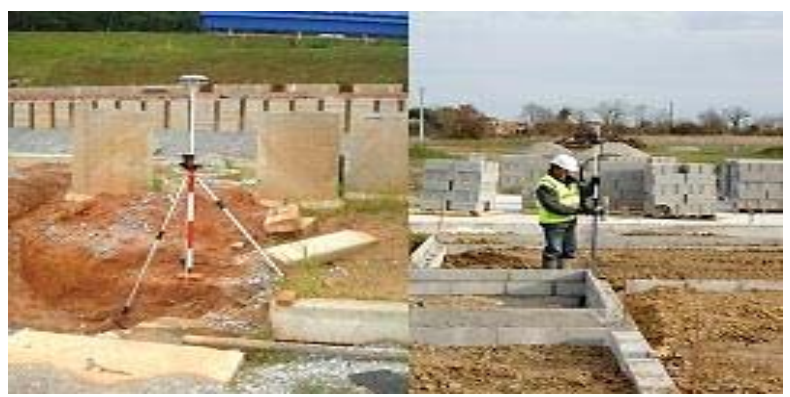

FIGURA 2: Exemplo de uso de sistema GNSS para locação em canteiro de obras. FONTE: RODRIGUES (2014).

\subsection{USO DE SCANNER A LASER}

O chamado Laser Scanner Terrestre (LST) tem tido seu uso incrementado para documentação gráfica e métrica de objetos, sendo uma técnica não-destrutiva e também não invasiva. Os lasers scanners são equipamento do campo de Sensoriamento Remoto usados para levantamento de dados sobre determinada superfície observada, oferecendo precisão e rapidez na taxa de aquisição (BELLIAN; KERANS; JENNETE, 2005). Com este equipamento obtêm-se dados de digitalização em 3D que podem ser importados para soluções de software comumente usadas, para reconstrução de acidentes, arquitetura, engenharia civil, construção, ciência forense, fabricação industrial e levantamento terrestre. Dimensões de distância, cálculos de área e volume, tarefas de análise e de inspeção e documentação podem, portanto, ser realizadas de forma rápida, precisa e confiável através de dados obtidos com laser scanner.

Para construção civil, um possível interesse de uso desta tecnologia é pela possibilidade de aplicação de varredura a laser no desenvolvimento do projeto As Built. Este projeto implica no levantamento das medidas existentes nas edificações, transformando em desenhos técnicos, todas as informações encontradas "in loco" que se relacionem com os projetos do edifício, tais como: instalações elétricas, hidráulicassanitárias, sistema de prevenção e combate à incêndio, níveis, etc.

McCarthy (2000) apresenta um caso desenvolvido em uma usina de Detroit onde uma 
pequena equipe de engenheiros fez o trabalho de desenvolvimento do projeto "as built", com a ajuda de um sistema de varredura a laser (Cyra Technologies), em metade do tempo que seria despendido caso fosse usado o processo tradicional (Figura 3). O processo tradicional, neste tipo de trabalho, seria realizado por meio do desenvolvimento de um modelo do edifício e auxílio de uma combinação de fotogrametria e esboços manuais feitos "in loco".

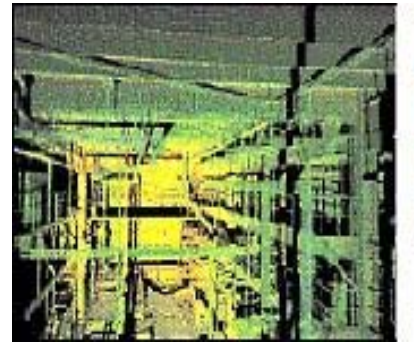

FIGURA 3: Pontos tridimensionais produzidos por scanner a laser para o interior de uma usina em DetroitEUA.

FONTE: MCCARTHY (2000).

\subsection{USO DE "RADIO FREQUENCY IDENTIFICATION" (RFID)}

Radio Frequency Identification é a tecnologia de identificação automática, na qual rádio frequência é utilizada para captura e transmitir dados. Para a construção, já em 1995, previa-se o uso em aplicações, tais como: operações de concretagem, codificação de custo de mão de obra e equipamentos e, também, controle de materiais (JASELSKIS et al., 1995). Com o surgimento do BIM (Modelagem da Informação da Construção), novas aplicações vão sendo desenvolvidas à medida que o novo padrão vai se consolidando (SUCCAR, 2009). Zhang et al. (2013) propõem uma investigação experimental para o uso de RFID integrado a modelagem BIM, para coletar informações visando o gerenciamento de facilidades e níveis de segurança durante o ciclo de vida de obras. A proposta prevê o uso de tags (etiquetas) de RFID permanentemente alocados em elementos da estrutura da obra, contendo informações de localização, entre outras. Durante a construção da edificação, os tags são temporariamente distribuídos nos espaços e aberturas, visando gerenciar diferentes níveis de riscos nas áreas de trabalho. Este é um exemplo prático, porém, a partir deste caso é possível vislumbrar a variedade de aplicações de RFID no canteiro de obras.

\section{CONSIDERAÇÕES FINAIS}

Descreve-se neste artigo a ideia de uso intensivo de recursos tecnológicos em canteiros de obras que, dentre outras ações, representam melhorias para o setor da Construção Civil. A TI somada a soluções em termos de metodologias, novos recursos e atividades inovativas, poderão significar em um real avanço na forma de se projetar e construir e, isto, tem sido estudado e conduzido por agentes setoriais de forma embasada no mercado.

Por outro lado, muitas vezes falta conhecimento aos gestores e aos técnicos para identificar e adotar novos recursos auxiliares ao andamento de processos de construção de edifícios. A maior dificuldade encontrada pelo setor para levar as soluções tecnológicas do escritório para o canteiro se dá devido a intervenientes, como: alto custo das tecnologias, desconhecimento da existência das ferramentas e formas de aplicação dos novos recursos tecnológicos, falta de interesse e de incentivo à capacitação profissional, etc.

A TI, no entanto, está presente em todos os segmentos e não será diferente na indústria da Construção Civil, uma vez que, como mostrado neste texto, podem-se fazer projeções reais de emprego destas tecnologias em grande escala em canteiros de obras, incorporando-as às atividades desde o planejamento até a execução. A partir do reconhecimento dos ganhos na aplicação de conceitos e recursos de $\mathrm{TI}$ a fim de facilitar a comunicação de soluções propostas no processo de planejamento, concretização e vivência diária de canteiros, a consolidação da aplicação destas novas tecnologias será uma consequência.

\section{REFERÊNCIAS BIBLIOGRÁFICAS}

ALVES, D. B. M.; ABREU, P. A. G. de; SOUZA, J. S. GNSS: Status, Modelagem Atmosférica e Métodos de Posicionamento. Revista Brasileira de Geometria,v. 1,n. 1. Universidade Tecnológica Federal do Paraná, Pato Branco, 2013. 8-13 p. 
AMIM, R. R. Realidade Aumentada aplicada à Arquitetura e Urbanismo. 120 p. Dissertação (Mestrado em Engenharia Civil) - COPPE, Universidade Federal do Rio de Janeiro, Rio de Janeiro, 2007.

APPLE. iPad in Business. 2013. Disponível em: http://www.apple.com/ipad/business/profiles/bechtel/ Acessado em: 24 de julho de 2014.

ASSOCIAÇÃO BRASILEIRA DE NORMAS TÉCNICAS. NBR 15575: edificações habitacionais: desempenho. Rio de Janeiro, 2013.

AZUMA, R. T. A Survey of Augmented Reality. Presence: Teleoperators and Virtual Environments, v.6, n.4, ago. 1997, p. 355-385.

AZUMA, M.; SILVESTRI, A. L. S.; FABRICIO, M. M. A Realidade Aumentada no Processo de Projeto Arquitetônico e Marketing das Edificações. In: III Simpósio Brasileiro de Qualidade do Projeto no Ambiente Construído \& VI Encontro de Tecnologia de Informação e Comunicação na Construção, 2013, Campinas, SP. Anais ..., 2013.

BALDAUF, J. P.; FORMOSO, C. T.; MIRON, L. I. G. Modelagem de Requisitos de Clientes de Empreendimentos Habitacionais de Interesse Social com o Uso de BIM. Ambiente Construído, Porto Alegre: ANTAC, v. 13, n. 3, p. 177-195, jul./set. 2013. Disponível em: http://seer.ufrgs.br/index.php/ambienteconstruido

/search/search. Acessado em: 10de março de 2014.

BECERIK-GERBER, B.; KENSEK, K. Building Information Modeling in Architecture, Engineering, and Construction: Emerging Research Directions and Trends. Journal of professional issues in engineering education and practice ASCE, v. 136, n. 3, 2010. p. 139147.

BEHZADAN, A.H.; TIMM, B.W.; KAMAT, V.R. GeneralPurpose Modular Hardware and Software Framework for Mobile Outdoor Augmented Reality Applications in Engineering. Advanced Engineering Informatics. Amsterdam, v. 22, n. 1, Jan.2008. p. 90-105. Disponível em: http://citeseerx.ist.psu.edu/viewdoc/summary?

doi=10.1.1.133.9408\&rank=1. Acessado em: 24 de julho de 2014.

BELLIAN, J. A.; KERANS, C.; JENNETTE, D. C. Digital Outcrop Models: applications of terrestrial scanning lidar technology in stratigraphic modeling. Journal of Sedimentary Research, v. 75, n. 2, p. 166-176, 2005.

FREITAS, M. R.; RUSCHEL, R. C. Aplicação de realidade virtual e aumentada em arquitetura. arquiteturarevista, v. 6, n. 2, p. 127-135, Jul-Dez. 2010. Disponível em: http://revistas.unisinos.br/index.php/

arquitetura/article/view/4553. Acessado em: 24 de julho de 2014.

JASELSKIS, E. et al. Radio-Frequency Identification Applications in Construction Industry. Journal of Construction Engineering and Management, 121(2), 1995. p. 189-196.
KIRNER, C. et al. Uso de Realidade Aumentada em Ambientes Virtuais de Visualização de Dados. In: Symposium on Virtual Reality - SVR 2004, 7, 2004, São Paulo. Anais... São Paulo. 2004. 112 p. CD ROM.

KÄHKÖNEN, K. Editorial: Virtual Reality Technology in Architecture and Construction. Electronic Journal of Information Technology in Construction ITcon, v. 8, 2003. p. 47-49. Disponível em: http://www.itcon.org/cgi-bin/works/Show?2003_8. Acessado em: 24 de julho de 2014.

NAKAMURA, A. Z.; GUIDARA JÚNIOR, P. Geotecnologias aplicadas à construção civil. Revista ENGENHARIA. ed. 601. 2010. p. 101-105. Disponível em: http://www.brasilengenharia.com.br/ed/601/art_const rucao_civil_601.pdf. Acessado em 24 de fevereiro de 2015.

MCCARTHY, D.C. Laser Scanner is a Model Helper for Construction Engineers. 2000. Disponível em: http://www. photonics.com/Article.aspx?PID $=5 \&$ VID $=14$ \&IID=54\&AID=5623. Acessado em: 24 de julho de 2014.

KRAINER, C. W. M. et al. Análise do impacto da implantação de sistemas ERP nas características organizacionais das empresas de construção civil. Ambiente Construído, Porto Alegre: ANTAC, v. 13, n. 3, p. 117-135, jul./set. 2013.

PAULA, N. de; UECHI, M. E.; MELHADO, S. B. Novas demandas para as empresas de projeto de edifícios. Ambiente Construído, vol.13, n.3. Porto Alegre: ANTAC. 2013.

RODRIGUES, D. Como Utilizar o seu GPS/GNSS RTK. 2014. LABTOPOPE - Laboratório Topográfico de Pernambuco: Universidade Católica de Pernambuco. Disponível em: http://www.labtopope.com.br/news/artigo-comoutilizar-o-seu-gps-gnss-rtk/. Acessado em: 24 de julho de 2014.

SHERMAN, W.R.; CRAIG, A.B. Understanding Virtual Reality. Interface, Application and Design. 1 ed. San Francisco: Morgan Kaufman Publishers - Elsevier Science, 2003. 582 p.

SUCCAR, B. Building Information Modelling Framework: a Research and Delivery Foundation for Industry Stakeholders. Automation in Construction. Netherlands: Elsevier Science, n. 18, 2009. p. 357-375.

TECHNE. Canteiro Digital. PINI, v. 182, maio 2012.

ZHANG, C. et al. Experimental Investigation of Using RFID Integrated BIM Model for Safety and Facility Management. In: INTERNATIONAL CONFERENCE ON CONSTRUCTION APPLICATIONS OF VIRTUAL REALITY, 13th. N. Dawood and M. Kassem (Eds.), 2013. Proceedings... October 2013, London. p. 150-159. 